\title{
Incidence de la procédure de prélèvement des sols fins sur les caractéristiques géotechniques mesurées en laboratoire
}

J.-C. BLIVET

Laboratoire régional des Ponts et Chaussées de Rouen, CETE

Normandie-Centre chemin de la Poudrière BP 245

76121 Le Grand-Quevilly Cedex

\section{LEVACHER}

\section{A. BAT}

UPRES A 6143 « Morphodynamique continentale et côtière »

Groupe de recherche génie civil, université de Caen 24, rue des Tilleuls 14032 Caen Cedex

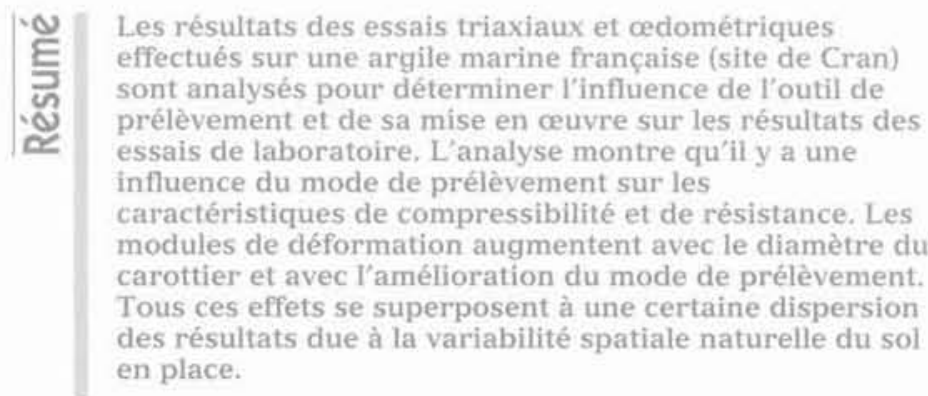

Les résultats des essais triaxiaux et œeiométriques ffectués sur une argite marine francruse (strede sont analysés pour déterminer l'influence de l'outil de prélèvement et de sa mise en cuvre sur les résultats des essais de laboratoire, L'analyse montre qu'il y a une modules de déformation augmentent avec le diamètre du cot avec lamélioration du mode de prélevement. des résultats due à la variabilité spatiale naturelle du sol en place.
NDLE : Les discussions sur cet article sont acceptées dans un délai de trois mois après sa parution.

\section{A large program of laboratory testing including triaxial} compression and cedometer test has been performed on french marine clay (in Brittany, Cran site). The main proposal of these investigations is to evaluate the influence of the sampling tools (type and procedure) on the laboratory tests results. The analysis of test results demonstrates that the sampling procedure

influences the resistance and the compressibility characteristics. The deformation modulus increases with the sampler diameter. as the improvement of the sampler procedure. Some effects are difficult to evaluate in front of the natural variability in space for the marine clay site. 


\section{Introduction}

Les études géotechniques nécessitent la connaissance des propriétés des sols concernés par les ouvrages. De nombreuses caractéristiques physiques, mécaniques ou hydrauliques des sols ne peuvent être obtenues qu'en effectuant des essais de laboratoire. Les paramètres ainsi mesurés dépendent, d'une part, de la qualité des prélèvements et, d'autre part, de la qualité des essais. La fiabilité de la valeur des paramètres est donc fortement influencée par les processus mis en cuvre depuis le carottage du sol sur le terrain jusqu'à linterprétation des résultats issus des essais.

La démarche d'assurance qualité actuellement en cours dans la plupart des laboratoires et bureaux d'étude repose sur un référentiel technique. Le respect des procédures écrites est une condition nécessaire pour atteindre la qualité. Mais parallèlement, il importe d'avoir un esprit critique et de bien comprendre les phénomènes mis en jeu.

Après avoir rappelé la classification des prélèvements en fonction des paramètres à mesurer, les caractéristiques des deux outils utilisés sont données : le carottier à piston stationnaire LPC et le carottier de gros diamètre de l'université Laval (Québec). La présente étude analyse le remaniement d'échantillons d'argile molle de Cran (Ille-et-Vilaine) prélevés avec ces deux types de carottiers. Cette étude a pour but d'analyser l'effet de l'outil de prélèvement et de sa mise en cuvre sur les paramètres mesurés de compressibilité, de déformabilité et de résistance. Les paramètres physiques, mécaniques et hydrauliques de cette arqile ont été mesurés au Laboratoire régional des ponts et chaussées de Rouen.

\section{2}

\section{Le prélèvement des sols fins cohérents}

\section{1}

\section{Les classes de prélèvement}

Pour un sol donné, la qualité du prèlèvement dépend de la technologie emplovée et de la compétence de l'équipe de sondages. Cependant de bons résultats obtenus sur des échantillons de bonne qualité ne donnent pas forcément une schématisation géotechnique correcte d'un site pour une étude. Il est nécessaire de prendre en compte la variabilité spatiale et le degré de représentativité des échantillons prélevés. La connaissance géologique locale ainsi que d'éventuelles études préalables permettent, en général, de faire le choix adéquat du programme de reconnaissance et des techniques de prélèvement.

Est qualifié d'intact, ou plutôt de non remanié, un prélèvement qui conserve, autant qu'on puisse l'apprécier, l'intégralité de la structure et des caractéristiques du sol, et sur lequel on peut effectuer des essais d'identification (teneur en eau, granulométrie, sédimentométrie, limites d'Atterberg...) ainsi que des essais mécaniques (triaxial, œdomètre...) permettant la prévision du comportement des ouvrages.
Pratiquement, aucun échantillon n'est véritablement intact car le prélèvement provoque toujours des modifications de l'état de contrainte et de déformation. Il est donc préférable de parler d'échantillon non remanié que l'on peut qualifier ainsi : échantillon qui, au laboratoire sous un chemin de contraintes approprié, fournit des résultats applicables à la prévision de la résistance en place, de l'amplitude et de la vitesse de déformation. Cela implique des déformations faibles au cours du carottage, déformations inférieures à celles obtenues lors de la rupture du sol (entre 1 et $4 \%$ pour la plupart des sols naturels) ; ainsi le sol naturel reste dans le domaine élastique réversible et les liens de diagenèse ne sont pas détruits (Fig. 1).

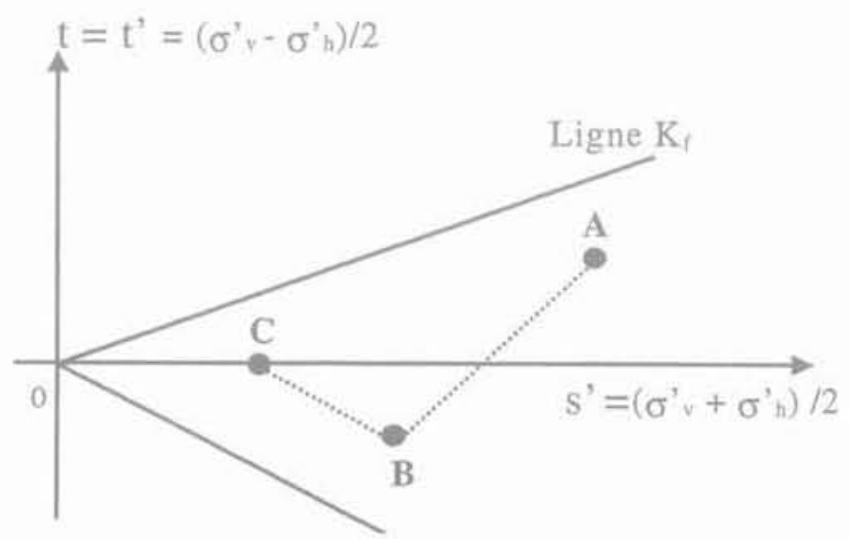

FIG 1 Modification schématique de l'état de contraintes effectives d'un échantillon de sol. A : état en place; B : état de l'échantillon dans le conteneur; $\mathrm{C}$ : état lors de la taille de l'éprouvette en laboratoire.

Effective stress path for a soil sample.

La qualité du prélèvement est fonction de l'usage que l'on veut faire de l'échantillon, c'est-à-dire de la nature des paramètres à mesurer nécessaires pour l'étude envisagée. Idel et al. (1969) ont proposé une classification pratique des prélèvements dans laquelle les échantillons sont attribués à une classe dite parfois de qualité allant de 1 à 5 , en fonction de leurs critères distinctifs typiques et des paramètres que l'on peut obtenir à partir du sol prélevé, Ces classes, reprises dans la norme sur les prélèvements XP P 94-202, se définissent à partir des caractéristiques géotechniques mesurables sur les échantillons. Le tableau I présente un résumé de cette classification.

TABLEAU Classification des prélèvements, (d'après Idel et al., 1969)

Samples classification

(after Idel et al., 1969)

\begin{tabular}{l|c|c|c|c|c} 
Classes & 1 & 2 & 3 & 4 & 5 \\
\hline $\begin{array}{l}\text { Paramètres } \\
\text { mesurables }\end{array}$ & Z, w, $\tau, \gamma_{\mathrm{d}}$ E, k & Z, , , w, k & Z, w & Z & $\begin{array}{c}\text { Échantillon } \\
\text { incomplet }\end{array}$ \\
\hline
\end{tabular}

avec les notions suivantes:

$\mathrm{Z}$ : Stratigraphie du sol ; $\tau_{\mathrm{r}}$ : Rêsistance au cisaillement; $w$ : Teneur en eau naturelle; $\gamma_{0}$ : Poids volumique du sol sec: $E$ : Module de déformation; $K$ : Coefficient de perméabilité.

Un échantillon " non remanié ») correspond à la classe 1; un échantillon "remanié ») est un échantillon de classe 3 ou 4. La différence entre la classe 3 et la classe 4 provient d'une perte de teneur en eau naturelle 
due au prélèvement, comme par exemple lors de prélèvement d'une tourbe avec les tarières continues. L'échantillon de classe 5 ne permet que d'identifier un changement de couche de sol. Cette classification est à utiliser une fois fixés les paramètres à mesurer. La classe de prélèvement souhaitée permet ensuite de choisir la meilleure adéquation possible entre la nature et l'état du sol et la technique du prélèvement à employer. La norme XP P 94-202 fournit des informations pour le choix des méthodes de prélèvement. La classe de prélèvement dépend donc essentiellement de l'objectif recherché, c'est-à-dire des paramètres de nature et d'état qui doivent être mesurés. Ainsi un prélèvement de classe 3 est un excellent échantillon pour effectuer une identification du sol et déterminer par exemple ses aptitudes au réemploi en remblais. La connaissance des lois comportement mécanique des sols nécessite l'utilisation d'échantillons de classe 1.

\section{$2 \Omega$}

\section{Recommandations pour le prélèvement de sols fins cohérents}

La diversité des procédés de prélèvement entraîne une très grande variété d'outils de prélèvement. Les prélèvements de sols fins de classe 1 s'effectuent essentiellement avec des carottiers poinçonneurs. Ceux-ci sont caractérisés par les paramètres liẻs aux dimensions des carottiers schématisés sur la figure 2 (diamètre extérieur, diamètre intérieur du carottier, longueur du tube, angle de coupe de la trousse...). Ils concernent principalement:
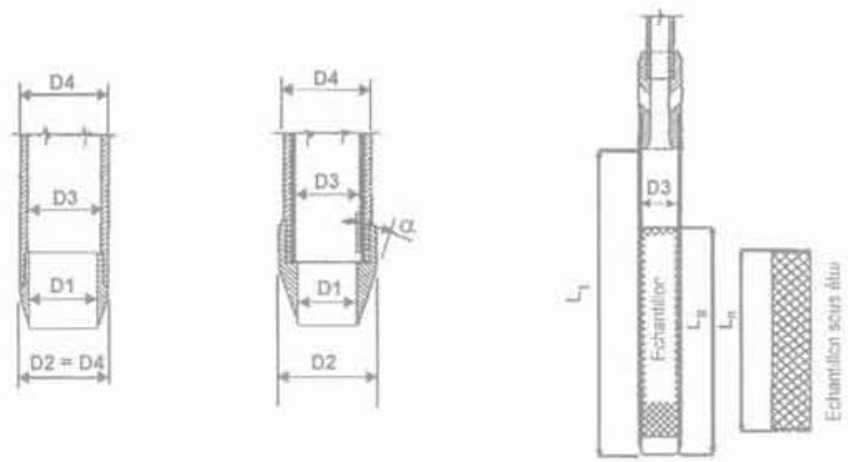

FG.2 Caractéristiques géométriques des prélèvements et des carottiers poinçonneurs. Geometric characteristics of thin-walled samplers.

- l'indice de surface $C_{\text {. }}$ est le rapport de la section droite de la trousse coupante du carottier à la section droite de la carotte, il représente approximativement le volume de sol déplacé par la paroi du carottier par rapport au volume de I'échantillon prélevé :

$$
C_{i=}=\frac{D_{2}^{2}-D_{1}^{2}}{D_{1}^{2}}
$$

- T'indice de jeu intérieur C relie le diamètre intérieur de la trousse coupante au diamètre intérieur du carottier, il permet de contrôler les actions du frottement lors du remplissage du carottier :

$$
C_{i}=\frac{D_{3}-D_{1}}{D_{1}}
$$

- l'indice de jeu extérieur $C_{0}$ contrôle le frottement extérieur, il relie le diamètre extérieur du carottier au diamètre extérieur de la trousse coupante :

$$
C_{0}=\frac{D_{2}-D_{4}}{D_{4}}
$$

$-\alpha$ est l'angle de biseau de la trousse, $c^{\prime}$ est l'angle d'attaque du carottier

- L, est la longueur utile du tube de carottage ;

$-L_{1} / D_{1}$ est le rapport de la longueur utile du tube de carottage au diamètre intérieur de la trousse ; c'est l'élancement maximal de l'échantillon.

Pour réaliser le prélèvement dans des conditions optimales, il est nécessaire de respecter les recommandations sur les dimensions des carottiers. Plus la paroi du carottier est mince, et plus l'angle de l'attaque de la trousse est petit, plus le remaniement est faible. Hvorslev (1949) a donné une valeur maximale de $15 \%$ à l'indice de surface $C_{\text {a }}$ et une valeur maximale de 10 degrés à l'angle $\alpha$. Lors du fonçage, la carotte subit une compression longitudinale. Le frottement latéral de la carotte à l'intérieur de l'étui peut être réduit par une faible différence entre le diamètre intérieur de la trousse et le diamètre intérieur de l'étui et par un élancement $L_{1} / D_{1}$ peu élevé. Le jeu intérieur $C$ est compris, en général, entre 0,5 et $1,5 \%$ et l'élancement est limité à 10. Pour réduire le frottement latéral sur la paroi extérieure, les carottiers peuvent avoir un diamètre extérieur de la trousse plus grand que le diamètre extérieur du tube carottier ; le diamètre du bossage antifriction est défini par l'indice du jeu extérieur $C_{c}$ qui peut atteindre 2 à $3 \%$ dans certains cas.

Les autres recommandations géométriques données par Hvorslev (1949) pour diminuer le remaniement sont les suivantes:

- un diamètre des échantillons $D_{1}: 75 \mathrm{~mm}$ pour les essais de routine, 100 à $150 \mathrm{~mm}$ pour les essais particuliers;

- le prélèvement par fonçage doit être réalisé rapidement et de facon continue, et l'échantillon de sol doit être préservé du contact de l'eau de forage.

En fait, malgré les précautions prises lors du prélèvement, il arrive que l'échantillon porte des traces de remaniement, principalement en parties haute et basse de la carotte et à la périphérie. L'essai en laboratoire se fait alors sur une partie médiane et interne de la carotte exempte de traces visibles de remaniement. Ceci impose un diamètre minimal de carottage en rapport avec les dimensions des échantillons d'essai. En pratique, pour une éprouvette d'essai de $70 \mathrm{~mm}$ de diamètre, on prélèvera au moins un échantillon de $80 \mathrm{~mm}$ pour permettre d'éliminer cette zone périphérique remaniée, avec une Iongueur maximale du carottier de l'ordre de $80 \mathrm{~cm}$.

Une étude réalisée sur des sols cohérents meubles prélevés avec des carottiers à piston stationnaire de différents diamètres $(50 \mathrm{~mm}, 63 \mathrm{~mm}, 80 \mathrm{~mm}$ et $100 \mathrm{~mm})$, a permis à Lemasson et al. (1975) de constater, en haut de la carotte, une diminution de la densité apparente qui peut aller jusqu'à $10 \%$ de la valeur moyenne et une diminution de la cohésion non drainée de $40 \%$ de sa valeur moyenne. Ils ont alors défini les limites des zones intactes le long des carottes prélevées, en précisant, en fonction de l'élancement du carottier et de son diamètre, les longueurs des zones remaniées en haut et en bas de la carotte. Ainsi, avec ces carottiers, une longueur minimale de quatre diamètres est remaniée en haut de la carotte et une longueur minimale d'un diamètre est remaniée en bas de la carotte. 


\section{Procédure de mise en œuvre du carottier à piston stationnaire (CPS)}

Le carottier à piston stationnaire, est le moyen de prélèvement des argiles molles le plus utilisé. Il est recommandé pour le prélèvement d'échantillons de classe 1, de sols fins de consistance molle à ferme (norme XP P 94-202, 1995). Les carottiers à piston stationnaire classiques ont un diamètre de $80 \mathrm{~mm}$ ou $100 \mathrm{~mm}$. Ce sont des carottiers à paroi mince constitués d'un tube carottier gainé intérieurement d'un étui en plastique, pour le conditionnement des échantillons, et dans lequel coulisse un piston. Ce dernier obture le carottier pendant son enfoncement jusqu'à la cote où doit commencer le carottage ; il assure en plus une étanchẻité en partie haute du prélèvement ce qui évite de perdre la carotte lors de la remontée de l'ensemble.

Pendant la phase de mise en place, le piston est verrouillé à la base du tube carottier, c'est ainsi que le carottier est descendu dans le trou de forage jusqu'à la cote de prélèvement (Fig. 3a).

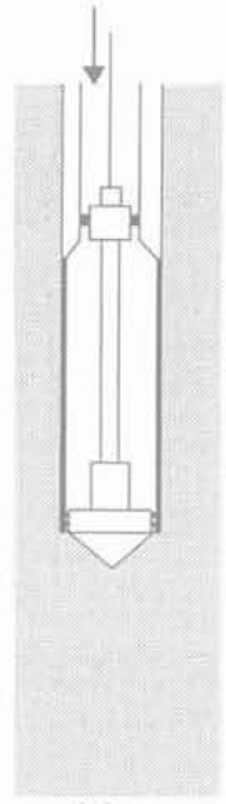

(a)

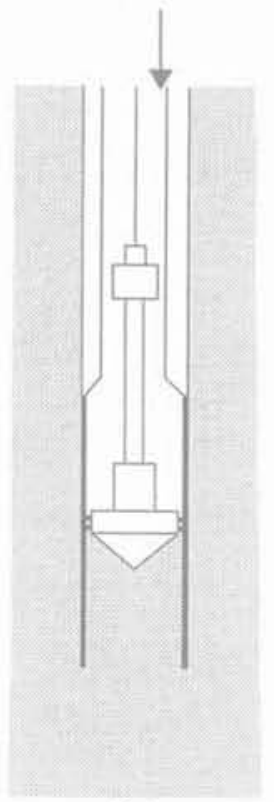

(b)

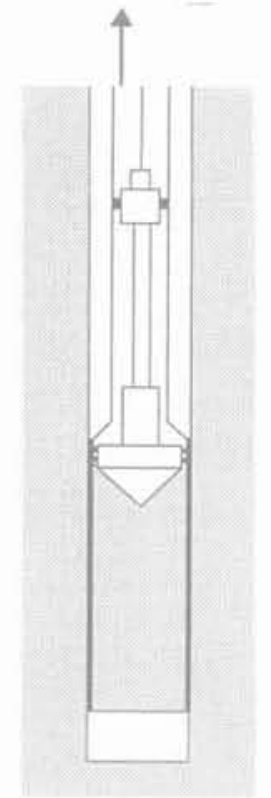

(c)
FIG:3 Phases de manœuvre du carottier à piston stationnaire.

Procedure stages of stationary piston sampler.

Cette technique permet d'éviter que du sol indésirable se retrouve à l'intérieur du carottier et permet de connaitre le niveau exact des prélèvements. Au début du carottage, le piston est désolidarisé du tube et est maintenu fixe, pendant que le carottier prélève le sol (Fig. 3b). Une fois la profondeur voulue atteinte, le piston et le tube carottier sont à nouveau solidarisés (Fig. 3c).

Après la remontée de l'ensemble du carottier à la surface, l'échantillon est extrait avec son étui. Une fois le prélèvement récupéré, il est numéroté, étiqueté et fermé hermétiquement avec un mélange de vaseline et de cire et des bouchons aux deux extrémités. Les étuis ainsi fermés sont ensuite placés verticalement dans des caisses habillées de mousse afin que le transport ne détruise pas le matériau par vibrations. Au laboratoire, au moment de l'essai, l'échantillon est extrait de l'étui en découpant le tube suivant deux génératrices.

\section{4}

\section{Procédure de mise en œuvre du carottier Laval (Laval 200)}

Le carottier de gros diamètre Laval 200, qui a été utilisé, est un carottier semblable en tout point à celui développé à l'université Laval au Québec (La Rochelle et al., 1981). Ce carottier permet de prélever des échantillons de sols cohérents de $200 \mathrm{~mm}$ de diamètre. Il est constitué de deux tubes: un tube intérieur, qui est l'outil de prélèvement, terminé à sa partie inférieure par une trousse coupante d'angle de biseau $5^{\circ}$, et un tube extérieur équipé à sa partie inférieure d'une couronne dentée qui permet de désagréger le sol autour du tube intérieur. Les deux tubes sont rendus solidaires lors de la descente du carottier au niveau du prélèvement et lors de la remontée de l'échantillon vers la surface.

Les opérations de prélèvement au moyen du carottier Laval sont réalisées comme suit :

- descente du carottier dans le forage jusqu'au niveau du prélèvement (Fig. 4a) ;

- désolidarisation des deux tubes intérieur et extérieur et fonçage du tube intérieur seul (Fig. 4b) :

- descente du tube extérieur entraîné en rotation jusqu'à ce que la base de la couronne dépasse l'extrémité de la trousse coupante d'environ $2 \mathrm{~cm}$. Cette rotation est accompagnée par l'injection du liquide de forage (en général de l'eau claire) entre le tube intérieur et le tube extérieur afin d'entrainer vers la surface l'argile désagrégée qui entoure le tube intérieur (Fig. 4c) :

- reverrouillage du carottier et fermeture étanche de lévent du tube intérieur puis remontée du carottier vers la surface (Fig. 4d),

Après remontée du carottier, l'échantillon est extrait du tube intérieur par extrusion sur le site puis découpé en morceaux de $20 \mathrm{~cm}$ de hauteur. Les morceaux sont placés sur des plaques de contre-plaqué « marine»,

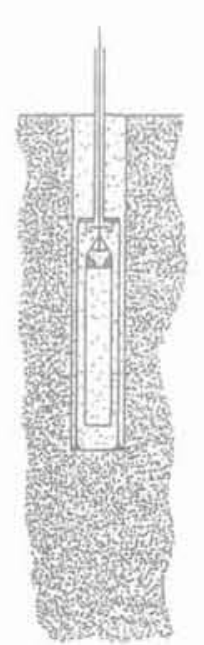

(a)

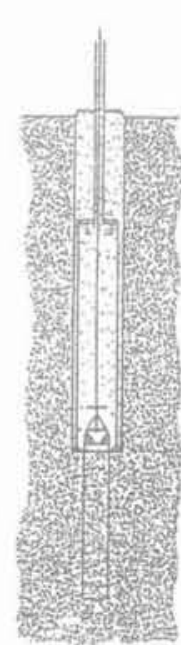

(b)

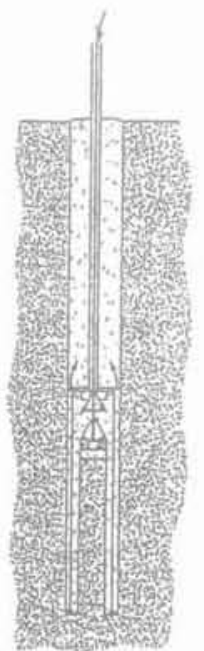

(c)

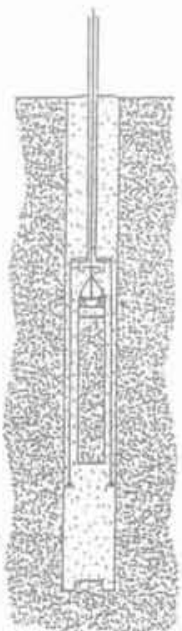

(d)
FG. 4 Principe de fonctionnement du carottier Laval (d'après La Rochelle et al., 1981). Procedure stages of Laval sampler. 
préalablement enduites de mélange de paraffine et recouvertes de papier film. Ensuite, les morceaux sont eux-mêmes enveloppés dans du papier film et recouverts d'un mélange de paraffine et d'huile de vaseline en trois couches minimum dans le but d'assurer une bonne étanchéité. Une fois étiquetés, les échantillons sont transportés dans des caisses où ils reposent sur de la mousse synthétique servant d'amortisseur contre les vibrations et les chocs.

\section{3}

\section{Incidence de la procédure de prélèvement}

\section{1}

\section{Caractéristiques générales du site de Cran}

Pour réaliser cette étude expérimentale le choix s'est porté sur le site du pont de Cran (franchissement de la Vilaine, en aval de Redon). A Cran, la rive droite est constituée par un dépòt récent d'argile molle marine de $17 \mathrm{~m}$ d'épaisseur reposant sur un substratum rocheux (schiste et phtanites). Les caractéristiques géotechniques moyennes des couches de sols identifiées dans le site de Cran sont indiquées dans le tableau II. L'argile de Cran contient environ 40 à $50 \%$ d'éléments inférieurs à $2 \mu \mathrm{m}$, la fraction fine étant surtout composée de montmorillonite, d'illite et de kaolinite en proportion plus faible. D'une manière générale, l'argile de Cran est grise et contient des fibres végétales (roseaux) presque toujours verticales.

\section{2}

\section{Le réalésage du forage}

Lors des prélèvements à l'aide du carottier à piston stationnaire CPS, nous avons constaté sur le chantier que du sol pénètre spontanément dans le carottier dès le déverrouillage du piston et ceci avant le début du fonçage. Durant la descente du carottier fermé par le piston jusqu'à la cote de la fin du prélévement précédent, le sol provenant des parois de forage est refoulé latéralement et comprimé sous le piston (Fig. 5a). Ceci risque d'entraîner plus bas les terrains supérieurs, déformant ainsi la stratification naturelle (Fig. 5b). Le déverrouillage du piston libère ce sol comprimé qui pénètre à l'intérieur du carottier (Fig. 5c).
Le sol qui pénètre ainsi spontanément dans le carottier a toutes les chances d'être remanié. Si on ne tient pas compte lors du fonçage de cette remontée initiale de sol, du sol intact carotté se trouve comprimé à l'intérieur du carottier lorsque, vers la fin du fonçage, le carottier est déjà plein. La carotte est alors remaniée par compression longitudinale, rendant ainsi incertaines les propriétés mécaniques mesurées. De plus, la phase finale du fonçage se poursuivant avec un carottier complètement rempli de sol, l'outil agit alors comme un gros pénétromètre sur les couches de sol sous-jacentes qui sont perturbées avant leur carottage (Fig. 5d).

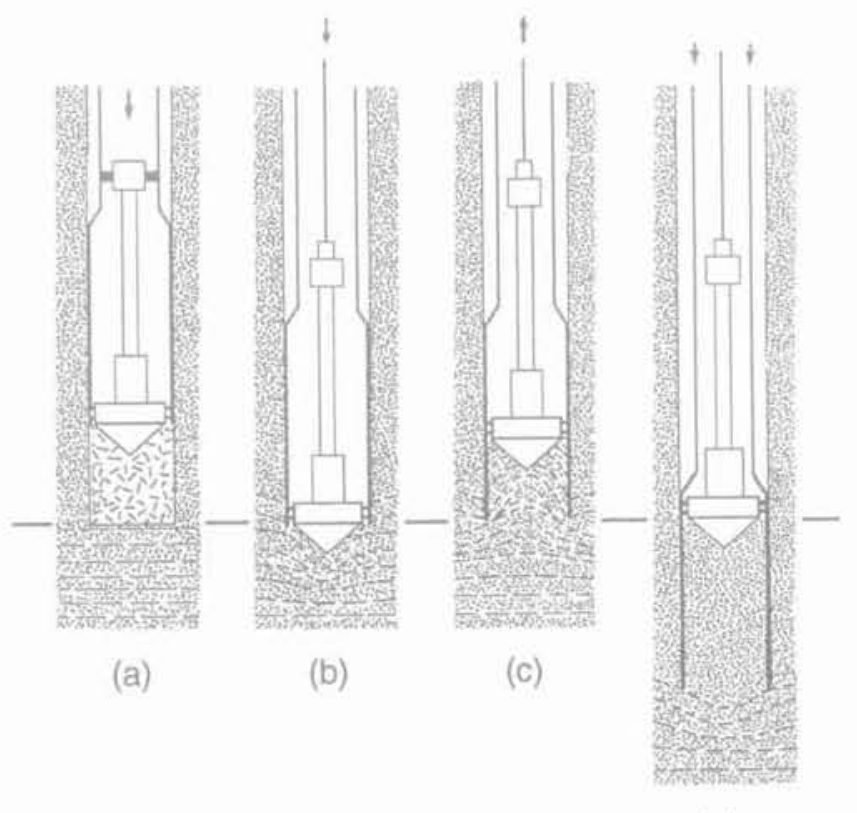

(d)

FiG.s Schéma explicatif du comportement du sol lors du prélèvement au carottier à piston stationnaire.

Comportment of soil during sampling with a stationary piston sampler.

Dans le cadre de travaux de recherche (Bat, 1999), nous avons relevé lors d'un sondage ( $\mathrm{K} 7$ ), avec le carottier CPS de diamètre $80 \mathrm{~mm}$, sur le site de Cran, les différentes hauteurs de sol pénétrant spontanément dans le carottier lors du déverrouillage du piston.

Cette hauteur du sol peut ètre supérieure à $10 \mathrm{~cm}$. Les plus grandes hauteurs ont été constatées en profondeur avec $16 \mathrm{~cm}$ à $6,80 \mathrm{~m}$. Sur ce site au-delà de $8,40 \mathrm{~m}$ le sol devient plus consistant, la remontée du sol est alors plus faible (Fig. 6).

TABtEAUIII Caractèristiques géotechniques moyennes du sol de Cran. Geotechnical properties of Cran site

\begin{tabular}{|c|c|c|c|c|c|c|c|c|c|c|c|}
\hline $\begin{array}{l}\text { Couche } \\
\text { Protondeur }\end{array}$ & $\begin{array}{l}\text { W } \\
(\%)\end{array}$ & $\left(w_{1}\right)$ & $I_{3}$ & 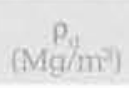 & e & $\begin{array}{l}\mathrm{MO} \\
(\%)\end{array}$ & $\frac{\mathrm{C}_{2}}{(\mathrm{kPa})}$ & $C_{8}$ & $\begin{array}{c}\sigma_{p}^{\prime} \\
(\mathrm{kPa})\end{array}$ & $\begin{array}{l}\sigma^{\prime} \\
(\mathrm{kPa})\end{array}$ & $\begin{array}{c}\mathrm{c}^{2}-\mathrm{b} \\
\text { (kPa-degré) }\end{array}$ \\
\hline${ }_{0}^{1}$ à $2 \mathrm{~m}$ & 48 & 75 & 30 & 1,10 & 1,3 & 1 & 45 & 0,45 & 160 & 7 & \\
\hline $\begin{array}{l}2 \\
2 \mathrm{a} 4 \mathrm{~m}\end{array}$ & 108 & 125 & 82 & 0,66 & 2,9 & 3 & 15 & 1,64 & 25 & 16 & $0,0-30$ \\
\hline $\begin{array}{l}3 \\
4 \text { à } 8 m\end{array}$ & 64 & 70 & 31 & 0,93 & 1,8 & 2 & 40 & 0.70 & 96 & 30 & \\
\hline $\begin{array}{l}4 \\
8 \mathrm{a} 17 \mathrm{~m}\end{array}$ & 73 & 87 & 47 & 0,91 & 1.7 & 4 & 39 & 0,85 & 105 & 67 & $0,0-34$ \\
\hline
\end{tabular}




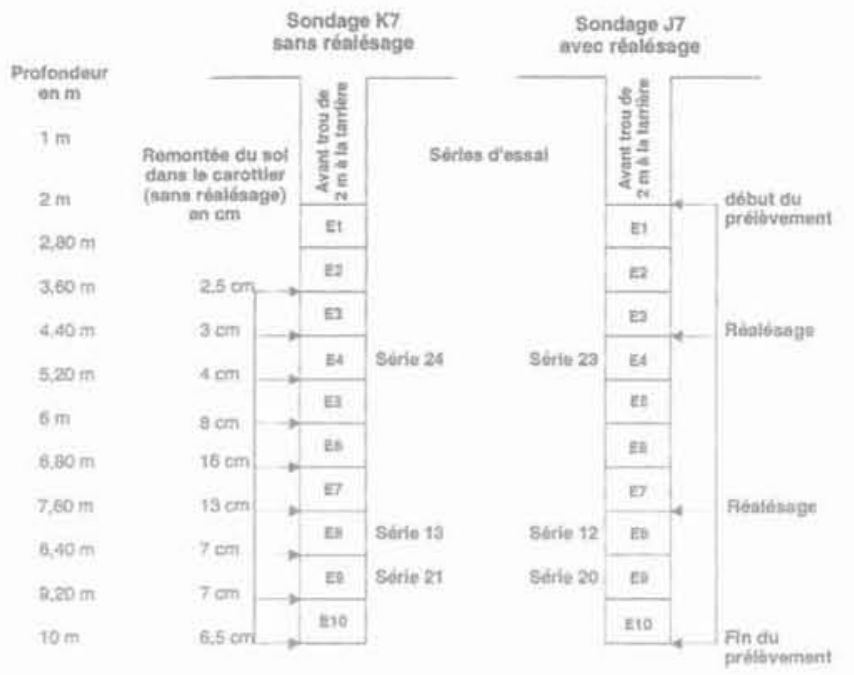

FIG. 6 Récapitulatif des remontées de sol et des séries d'essais dans les sondages au CPS avec et sans réalésage.

Effect of sampling procedure with a stationary piston sampler:

Pour éviter cette remontée de sol dans le carottier avant le début de fonçage, nous proposons de réaliser les sondages au carottier CPS, en procédant au préalable à un réalésage à la tarière, afin de nettoyer le trou de forage du sol indésirable et de s'assurer avec précision des niveaux de prélèvement des carottes. La figure 7 montre les différentes étapes à suivre afin de prélever le sol dans les meilleures conditions:

Après un prélèvement, les pertes géométriques provenant des parois encombrent le trou de forage (Fig. 7 a). On procède alors au réalésage à la tarière de $152 \mathrm{~mm}$ de diamètre (Fig. 7b). Le forage est alors prêt pour le prélèvement (Fig. 7c). A noter que pour éviter les gradients hydrauliques importants qui viendraient perturber le sol en fond de forage, il importe de maintenir un niveau d'eau dans le forage identique au niveau de la nappe.

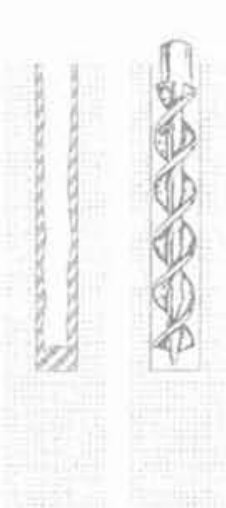

(a)

Phases du réalésage

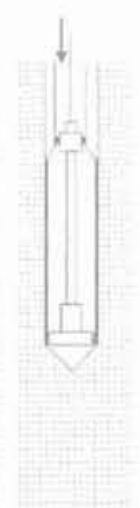

(d) (e)

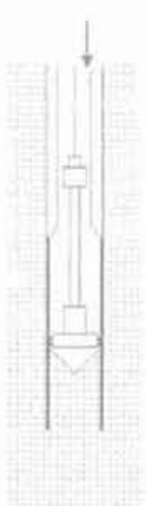

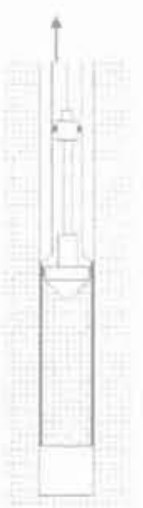

(f)
Phases de mise en oeuvre du carottler

HG.7 Les différentes étapes avec réalésage à la tarière lors du carottage au CPS.

Procedure stages of a stationary piston sampler with a clean-out auger.

\section{Effet de la méthodologie de mise en œuvre du carottage}

Dans le but de voir si le réalésage du forage, réduisant la remontée spontanée du sol lors du déverrouillage du piston, améliore la qualité des prélèvements avec le carottier à piston stationnaire (CPS 80), on a effectué sur le site de Cran différentes séries d'essais sur des échantillons prélevés dans les deux sondages K7 (sans réalésage) et J7 (avec réalésage). Les différentes séries d'essais ont été réalisées sur des prélèvements qui avoisinent la cote où a été effectué le réalésage (Fig. 6). Les séries 23, 24, 12 et 13 englobent des essais cedométriques dans la partie haute et la partie basse de la carotte et des essais UU. Les séries 20 et 21 comportent des mesures de cohésion au scissomètre de laboratoire, le long des carottes (échantillons E9, des deux sondages K7 et J7. Fig. 6). Les résultats de ces séries d'essais permettent de comparer la variation des paramètres de résistance et de compressibilité dans les deux types de sondages (avec ou sans réalésage).

Pour caractériser la déstructuration des échantillons d'argile molle de Cran, due à la mise en cuvre du carottier, on utilise les paramètres suivants :

- la déformation verticale sous le poids des terres $\varepsilon_{v=}($ en $\%)$ à l'cedomètre donnée par la formule (4) avec e l'indice des vides initial de l'éprouvette et e l'indice sous le poids des terres. C'est la déformation de l'échantillon prélevé, lorsque la contrainte verticale en place lui est à nouveau appliquée. Plus le remaniement est grand plus la valeur de ce paramètre sera élevée. A l'Institut norvégien de géotechnique (Lacasse et Berre, 1988), des déformations $\varepsilon$ inférieures à $2 \%$ attestent de la bonne qualité des échantillons d'argiles molles prélevés:

$$
\varepsilon_{\text {vo }}=\frac{e_{j}-e_{0}}{e_{i}}
$$

- la cohésion non drainée au scissomètre de laboratoire $\mathrm{c}_{\text {us }}($ en $\mathrm{kPa})$

- la déformation de rupture $\varepsilon$, (en \%), elle augmente avec le remaniement et peut servir d'indice de la qualité des échantillons. Des déformations $\varepsilon_{\text {, }}>4 \%$ signifient que les échantillons sont de qualité moindre ;

- la cohésion non drainée $c_{u}(e n k P a)$ diminue avec le remaniement ;

- le module de déformation $\mathrm{E}_{50}$ (en $\mathrm{MPa}$ ) représente le module sécant à la mi-rupture. Il diminue avec le remaniement et il est plus indicatif et plus sensible à l'effet du remaniement que les critères de résistance $\left(\varepsilon_{p}, c_{j}\right)$. Les critères $\varepsilon_{p} E_{c n}$ et $c$ sont déterminés à l'issue des essais à l'appareil triaxial non drainé non consolidé (UU).

\section{$3: 34$}

\section{Incidence sur les paramètres de compressibilité et de consolidation}

Le récapitulatif des principales caractéristiques initiales des éprouvettes cedométriques des différentes séries 23, 24, 12 et 13 est donné dans le tableau III. L'analyse de ces données conduit aux observations sui- 
TABLEAU III Caractéristiques initiales des éprouvettes œdométriques des séries 12, 13, 23 et 24 Initial characteristics for oedometric samples

\begin{tabular}{|c|c|c|c|c|c|c|c|}
\hline \multirow{2}{*}{ Carottier } & \multirow{2}{*}{ Sêrie } & \multirow[b]{2}{*}{$\begin{array}{c}\text { Qdométre } \\
n^{*}\end{array}$} & \multicolumn{5}{|c|}{ Caractéristiques initiales } \\
\hline & & & $\begin{array}{l}\text { Profondeur } \\
\text { (m) }\end{array}$ & $\begin{array}{c}\sigma_{\mathrm{y}}^{\prime} \\
(\mathrm{kPa})\end{array}$ & $\frac{p}{\left.f g^{\prime} / m^{2}\right)}$ & $\begin{array}{l}W \\
(\%)\end{array}$ & $e_{i}$ \\
\hline Avec réalésage & $\begin{array}{l}23 \\
23 \\
12 \\
12\end{array}$ & $\begin{array}{r}64 \text { (Haut) } \\
65 \text { (Bas) } \\
47 \text { (Haut) } \\
46 \text { (Bas) }\end{array}$ & $\begin{array}{l}4,5 \\
4,9 \\
7,8 \\
8,3\end{array}$ & $\begin{array}{l}28 \\
30 \\
45 \\
48\end{array}$ & $\begin{array}{l}1.5 \\
1.4 \\
1.5 \\
1.4\end{array}$ & $\begin{array}{r}91 \\
110 \\
84 \\
104\end{array}$ & $\begin{array}{l}2,3 \\
2,8 \\
2,1 \\
2,7\end{array}$ \\
\hline Moyenne & 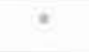 & 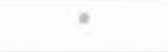 & 6,4 & 38 & 1,4 & 97 & 2,5 \\
\hline Sans réalésage & $\begin{array}{l}24 \\
24 \\
13 \\
13\end{array}$ & $\begin{array}{r}66 \text { (Haut) } \\
67 \text { (Bas) } \\
49 \text { (Haut) } \\
48 \text { (Bas) }\end{array}$ & $\begin{array}{l}4,5 \\
5,0 \\
7,7 \\
8,2\end{array}$ & $\begin{array}{l}28 \\
31 \\
45 \\
47\end{array}$ & $\begin{array}{l}1,5 \\
1,5 \\
1,4 \\
1,5\end{array}$ & $\begin{array}{l}85 \\
77 \\
64 \\
93\end{array}$ & $\begin{array}{l}2,2 \\
1,9 \\
2,0 \\
2,3\end{array}$ \\
\hline Moyenne & - & * & 6,4 & 38 & 1,5 & 80 & 2,1 \\
\hline
\end{tabular}

vantes: l'indice des vides e pour le sondage avec réalésage varie entre 2,1 et 2,8 , pour des teneurs en eau variant entre 84 et $110 \%$ alors que l'indice des vides du sondage non réalésé varie entre 1,9 et 2,3 pour des teneurs en eau variant entre 64 et $93 \%$. Ces données montrent une dispersion spatiale naturelle assez élevée sur le site.

Dans les deux sondages, on constate des écarts de teneur en eau de plus de 20 points à même profondeur, ces écarts attestent d'une différence de comportement et d'une variabilité naturelle du massif argileux de Cran. En effet les argiles molles de Cran ont une histoire source de discontinuité et d'hétérogénéité. Ces discontinuités sont liées au processus de sédimentation et de consolidation et aux mouvements subit par les terres lors de l'évolution des estuaires et des creusements des cours d'eau. Cette hétérogénéité permet d'expliquer que des échantillons prélevés à la même profondeur ont des propriétés physiques et mécaniques différentes.

Néanmoins, en dépit de la dispersion naturelle des caractéristiques du sol en place, nous constatons un effet du réalésage du forage sur la qualité des échantillons prélevés. En comparant des éprouvettes de même état initial, le prélèvement avec réalésage donne des déformations sous le poids des terres $\varepsilon_{v p}$ plus faible (tableau IV : œdomètres 64 et 66 de déformations respectives 1,1 et $3,5 \%$ ) ; cela indique que le réalésage du forage améliore la qualité des prélèvements.

L'examen des courbes normalisées de compressibilité des essais œdométriques œdomètre 64 (avec réalésage) et œdomètre 66 (sans réalésage) montre que l'absence de réalésage modifie l'état du sol dans la carotte. Le sol dans le haut de la carotte des prélèvements sans réalésage a subi lors du carottage une forte compression, suivie d'un relâchement après déverrouillage du piston. Lorsqu'on désolidarise le piston du carottier avant fonçage, les couches de sol comprimées sous le piston et qui proviennent des parois du forage remontent dans le carottier, ceci constitue la partie haute de l'échantillon fortement remaniée. Ce sol, provenant de couches plus élevées, comparé au sol qui est prélevé à des niveaux plus bas accuse un état de surconsolidation plus faible. On constate un écart de $\sigma^{\sigma}$ de $37 \%$ entre les œdomètres 64 et 66 (respectivement $\sigma_{p}=78 \mathrm{kPa}$ et $\sigma_{\mathrm{p}}=49$, tableau IV).

D'autre part, dans le domaine normalement consolídé et précisément pour la contrainte $\sigma^{\prime}=100 \mathrm{kPa}$, la comparaison des deux modules cedométriques de l'œdomètre 64 (avec réalésage) et l'cedomètre 66 (sans réalésage) montre un écart entre les modules. $\mathrm{E}_{\text {ou }}$ de $45 \%$ (respectivement $E_{c r d}=1814 \mathrm{MPa}$ et

TABLEAUIV Effet du réalésage sur les paramètres de compressibilité et de consolidation (séries : 12, 13, 23 \& 24) Effect of clean procedure on compressibility and consolidation properties of samples (series 12, 13, 23 \& 24)

\begin{tabular}{|c|c|c|c|c|c|c|c|c|c|c|}
\hline \multirow[b]{2}{*}{ Carottier } & \multirow{2}{*}{ Série } & \multirow[b]{2}{*}{$\begin{array}{l}\text { Cedomètre } \\
\text { na }^{\circ}\end{array}$} & \multicolumn{8}{|c|}{ Paramètres de compressibilité et de consolidation } \\
\hline & & & $C_{2}$ & $\mathrm{C}_{\mathrm{c}}$ & $e_{0}$ & $\begin{array}{c}\sigma \\
(\mathrm{KPa})\end{array}$ & E & $\begin{array}{l}\mathrm{E}_{\text {add }} \\
\text { (MPa) }\end{array}$ & $\mathrm{B}_{\mathrm{oc}}$ & $\left(\times 10^{-1} \mathrm{~m}^{2} / \mathrm{s}\right)$ \\
\hline Avec réalésage & $\begin{array}{l}23 \\
23 \\
12 \\
12\end{array}$ & $\begin{array}{r}64 \text { (Haut) } \\
65 \text { (Bas) } \\
47 \text { (Haut) } \\
46 \text { (Bas) }\end{array}$ & $\begin{array}{l}0,02 \\
0,05 \\
0,02 \\
0,04\end{array}$ & $\begin{array}{l}1,1 \\
1,5 \\
1,0 \\
1,3\end{array}$ & $\begin{array}{l}2,3 \\
2,8 \\
2,1 \\
2,6\end{array}$ & $\begin{array}{l}78 \\
64 \\
68 \\
55\end{array}$ & $\begin{array}{l}1,1 \\
1,1 \\
2,8 \\
6,3\end{array}$ & $\begin{array}{r}1814 \\
905 \\
1351 \\
857\end{array}$ & $\begin{array}{l}2,8 \\
2,3 \\
1,5 \\
1,2\end{array}$ & $\begin{array}{r}10 \\
4 \\
19 \\
5\end{array}$ \\
\hline Moyenne & * & $=$ & 0,03 & 1,2 & 2,5 & 66 & 2,8 & 1232 & 1,9 & 10 \\
\hline Sans réalésage & $\begin{array}{l}24 \\
24 \\
13 \\
13 \\
\end{array}$ & $\begin{array}{r}66 \text { (Haut) } \\
67 \text { (Bas) } \\
49 \text { (Haut) } \\
48 \text { (Bas) }\end{array}$ & $\begin{array}{l}0,02 \\
0,01 \\
0,02 \\
0,04 \\
\end{array}$ & $\begin{array}{l}0,9 \\
0,9 \\
0,9 \\
1,2 \\
\end{array}$ & $\begin{array}{l}2,2 \\
1,9 \\
1,9 \\
2,2 \\
\end{array}$ & $\begin{array}{r}49 \\
139 \\
61 \\
75 \\
\end{array}$ & $\begin{array}{l}3,5 \\
1,9 \\
5,4 \\
3,0 \\
\end{array}$ & $\begin{array}{r}988 \\
4855 \\
1456 \\
1577 \\
\end{array}$ & $\begin{array}{l}1,8 \\
4,5 \\
1,4 \\
1,6\end{array}$ & $\begin{array}{r}4 \\
20 \\
3 \\
4 \\
\end{array}$ \\
\hline Moyenne & * & * & 0,02 & 1,0 & 2,1 & 81 & 3,5 & 2219 & 2,3 & 8 \\
\hline
\end{tabular}


$\sigma_{v}^{\prime}(\mathrm{kPa})$

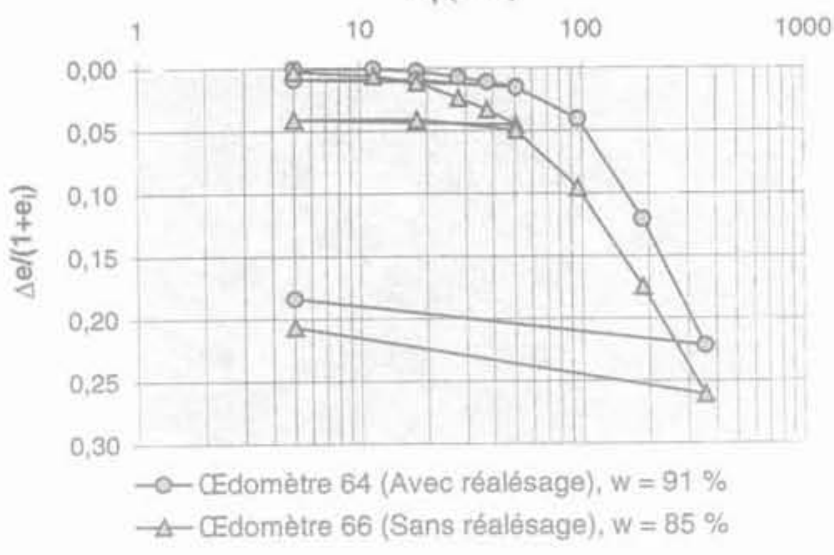

คiG. 8 Effet du réalésage sur les courbes de compressibilité.

Effect of clean procedure on compressibility curves.

$E_{\text {mid }}=988 \mathrm{MPa}$ ). Finalement, l'absence du réalésage entraine un état de déstructuration visible qui se traduit par des modules odométriques faibles, des déformations sous le poids des terres élevées et des contraintes de préconsolidation sous-estimées.

\section{Bengy}

\section{Incidence sur les paramètres de résistance au cisaillement}

L'effet du réalésage sur les paramètres de rigidité et de résistance a été analysé suivant deux démarches. La première consiste à comparer les résultats des essais UU réalisés sur des éprouvettes qui avoisinent la cote du réalésage (séries 12,13, 23 et 24 de la figure 6) ; la deuxième démarche consiste à comparer le long de deux carottes issues des sondages avec réalésage et sans réalésage les valeurs de la cohésion non drainée au scissomètre de laboratoire.

Les résultats d'essais UU à lappareil triaxial, réalisés sur les prélèvements avec et sans réalésage (respectivement J7 et K7), sont regroupés dans le tableau V. Les différentes éprouvettes présentent un même état de rupture à savoir une rupture en plan de cisaillement franc.
L'examen du tableau $V$ semble confirmer que le réalésage du forage, avant le fonçage du carottier, améliore la qualité des prélèvements. Les éprouvettes de sol issues du sondage avec réalésage donnent des modules de déformations moyens plus élevés, de l'ordre du double $\left(E_{50}=8 \mathrm{MPa}\right.$ et $\left.E_{50}=4 \mathrm{MPa}\right)$ et des déformations

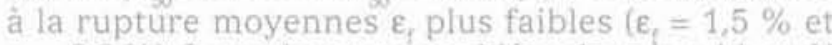
$\varepsilon_{i}=2,6 \%$ ). Les valeurs rassemblées dans le tableau $V$ montrent que la résistance au cisaillement varie peu pour cette sélection d'éprouvettes. Néanmoins l'effet du rẻalèsage sur la cohésion non drainée et sur les paramètres déformabilité peut être vu en comparant des éprouvettes de même état initial (même teneur en eau initiale). On constate alors qu'en l'absence du réalésage, la résistance au cisaillement co ainsi que le module déformation $E_{50}$ sont plus faibles et, que la déformation à la rupture est plus importantes (exemple: S23 UU2 et S24 UU2 avec respectivement $\varepsilon_{\mathrm{r}}=1,7 \%$ et $\varepsilon_{\mathrm{r}}=3,6 \%$ ).

Des séries d'essais au scissomètre de laboratoire (séries 20 et 21, Fig. 6) ont été réalisées, sur des prélèvements issus des deux sondages (réalésé et non réalésé) espacés de $2,50 \mathrm{~m}$. Ces mesures ont été effectuées tous les $10 \mathrm{~cm}$ sur le matériau contenu dans son étui, en évitant ainsi les inconvénients qui peuvent résulter des différentes opérations d'extraction et de taille des éprouvettes. La comparaison des profils de teneur en eau (Fig. 9a) montre une différence de teneur en eau moyenne entre les deux carottes de l'ordre de 20 points ; ceci correspond à la dispersion spatiale sur le site et permet de constater que des échantillons prélevés à la même profondeur ont des propriétés physiques différentes et donc des comportements mécaniques différents. Néanmoins, la figure 9b montre un effet du réalésage sur la résistance : on remarque que le prélèvement avec réalésage (série 20) montre une faible variation de la cohésion scissométrique le long de la carotte : le rapport de la cohésion centrale (au milieu de la carotte) sur les valeurs des cohésions le long de la carotte varie entre 0,7 et 1,1 pour cette série réalésée et dans une fourchette plus élevée pour la série 21 non réalésée, entre 0,2 et 1,4. La différence de la cohésion moyenne entre les deux carottes est attribuée à la différence des teneurs initiales et donc à une différence du comportement du sol. En comparant les hauts des deux prélèvements s'avérant avoir un même état initial (même teneur en eau initiale), on constate que le réalé-

TABlEaUV Résultats des essais UU CPS 80 (Avec et sans réalésage) UU Tests results (with and without cleaning)

\begin{tabular}{|c|c|c|c|c|c|c|c|c|}
\hline Échantillon & $\begin{array}{l}\text { Profondeur } \\
\text { fmy }\end{array}$ & $\begin{array}{l}w \\
(\%)\end{array}$ & $\begin{array}{c}\rho \\
(\mathrm{Mg} / \mathrm{m})\end{array}$ & $\begin{array}{c}\varepsilon \\
(\%)\end{array}$ & $(\mathrm{KPa})$ & $\begin{array}{l}\varepsilon_{m} \\
(\%)\end{array}$ & $\frac{E_{i t}}{|M P a|}$ & \\
\hline $\begin{array}{l}\mathrm{S} 23 \mathrm{UU} 3 \\
\mathrm{~S} 23 \mathrm{UU} 2 \\
\mathrm{~S} 12 \mathrm{UU2} \\
\mathrm{S} 12 \mathrm{UU} 3\end{array}$ & $\begin{array}{l}4,7 \\
4,8 \\
8,2 \\
8,2\end{array}$ & $\begin{array}{l}81 \\
99 \\
92 \\
92\end{array}$ & $\begin{array}{l}1,49 \\
1,46 \\
1,47 \\
1,47\end{array}$ & $\begin{array}{l}0,8 \\
1,7 \\
1,8 \\
1,8\end{array}$ & $\begin{array}{l}12 \\
18 \\
31 \\
45\end{array}$ & $\begin{array}{l}0,1 \\
0,4 \\
0,5 \\
0,5\end{array}$ & $\begin{array}{r}11 \\
4 \\
6 \\
9\end{array}$ & \multirow[t]{2}{*}{$\begin{array}{l}\text { Avec } \\
\text { réalésage } \\
\text { (J7) }\end{array}$} \\
\hline Moyenne & 6,5 & 91 & 1,47 & 1,5 & 27 & 0,4 & 8 & \\
\hline $\begin{array}{l}\text { S24 UU4 } \\
\text { S24 UU2 } \\
\text { S24 UU1 } \\
\text { S13 UU3 } \\
\text { S13 UU2 }\end{array}$ & $\begin{array}{l}4,7 \\
4,9 \\
5,0 \\
7,8 \\
7,9\end{array}$ & $\begin{array}{r}118 \\
102 \\
115 \\
105 \\
92\end{array}$ & $\begin{array}{l}1,36 \\
1,45 \\
1,41 \\
1,44 \\
1,44\end{array}$ & $\begin{array}{l}2,3 \\
3,6 \\
3,6 \\
2,2 \\
2,8\end{array}$ & $\begin{array}{l}23 \\
12 \\
25 \\
22 \\
43\end{array}$ & $\begin{array}{l}0,4 \\
0,6 \\
0,9 \\
0,6 \\
0,7\end{array}$ & $\begin{array}{l}5 \\
2 \\
3 \\
4 \\
6\end{array}$ & \multirow[t]{2}{*}{$\begin{array}{l}\text { Sans } \\
\text { réalèsage } \\
\text { (K7) }\end{array}$} \\
\hline Moyenne & 6,1 & 106 & 1,42 & 2,9 & 25 & 0,6 & 4 & \\
\hline
\end{tabular}




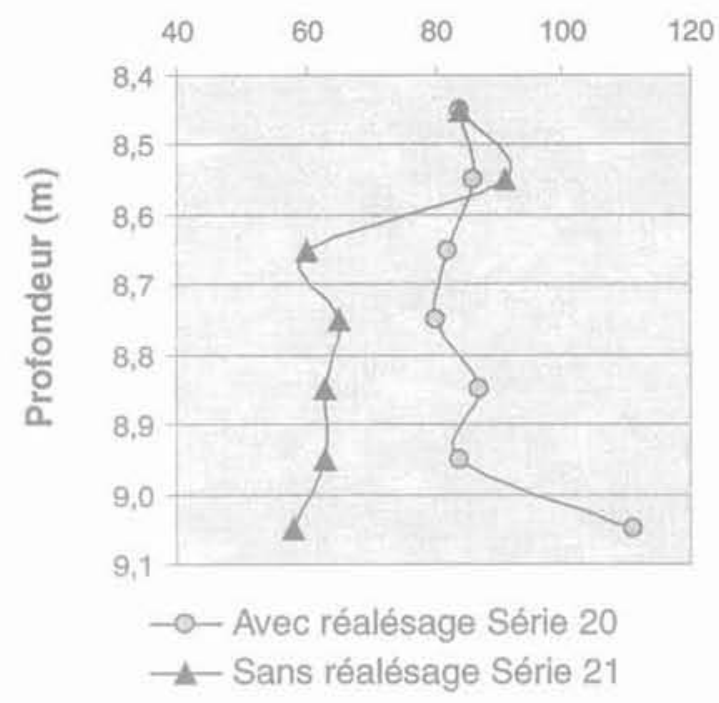

(a)

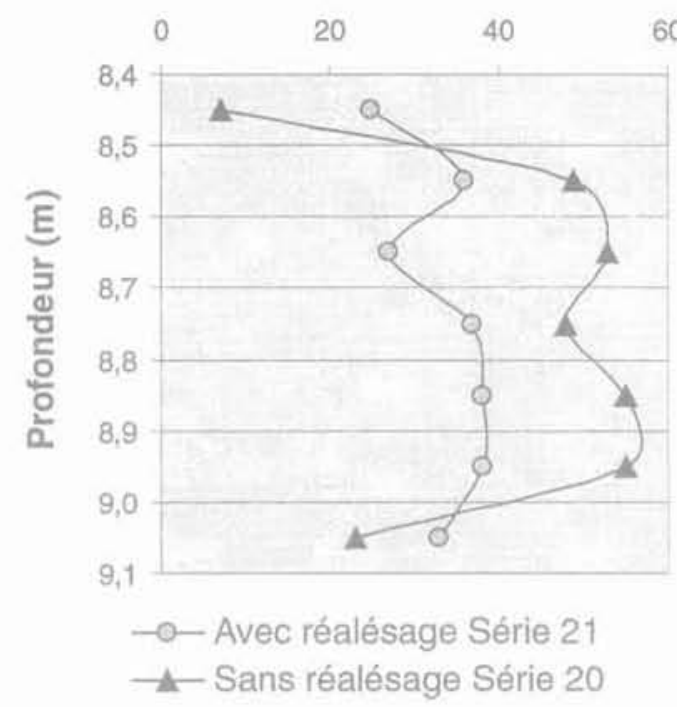

(b)

FiG.9. Variation de la teneur en eau et de la cohésion scissométrique le long des carottes dans les sondages réalésé et non réalisé.

Water content and undrained cohesion (vane test).

sage donne une cohésion scissométrique plus grande et donc une meilleure qualité.

On constate donc une influence de la mise en œuvre du carottage au piston stationnaire sur la compressibilité du sol et donc sur la qualité globale des prélèvements. Ces conclusions seraient vraisemblablement encore plus marquées si le réalésage avait été effectué avant chaque carottage, ce qui n'est pas le cas sur ces prélèvements (Fig. 6).

\section{4}

\section{Comparaison des carottiers cps et Laval 200}

Dans le but d'étudier l'́nfluence de l'outil de prélèvement sur les paramètres de résistance et de rigidité, des essais UU à l'appareil triaxial ont été réalisés sur des échantillons prélevés sur le site de Cran, par les deux carottiers ; le CPS 80 et le carottier Laval 200.

Les variations des principaux paramètres de résistance et de rigidité $\varepsilon_{r,} c_{u}$ et $E_{s y}$ dans le sondage Laval 200 et le CPS 80 (Fig. 10) permettent d'évaluer la déstructuration par effet de remaniement dủ à l'outil de prélèvement. On constate, en profondeur, un écart moyen du module de déformation $\mathrm{E}_{50}$ de plus de $50 \%$ (Fig. 10c), entre le CPS 80 et le Laval 200. Les valeurs moyennes des déformations en profondeur sont plus faibles (Fig. 10a) : pour le Laval $200\left(\varepsilon_{f}=1,7 \%\right.$ à $8 \mathrm{~m}$ de profondeur) comparé au CPS $80\left(\varepsilon_{\mathrm{f}}=2,6 \%\right.$ à $8 \mathrm{~m}$ de profondeur). La figure $10 \mathrm{~b}$ indique un léger écart entre la cohésion moyenne dans les deux sondages : la cohésion moyenne non drainée $\mathrm{c}_{\mathrm{u}}$ des échantillons Laval 200 est plus élevée que les valeurs moyennes du CPS 80 , mais dans une faible proportion (Fig. 10b). Ces résultats indiquent globalement que le carottier Laval 200 donne des échantillons de meilleure qualité que le carottier CPS 80. Ce sont surtout les modules de déformation qui sont affectés, les valeurs moyennes allant pratiquement du simple au double suivant le type de carottier.

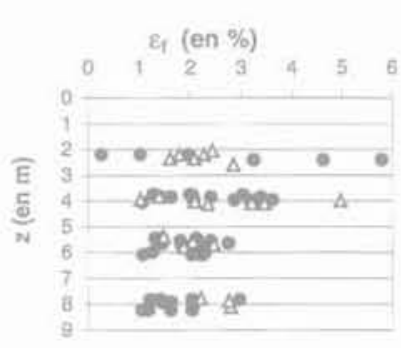

(a)

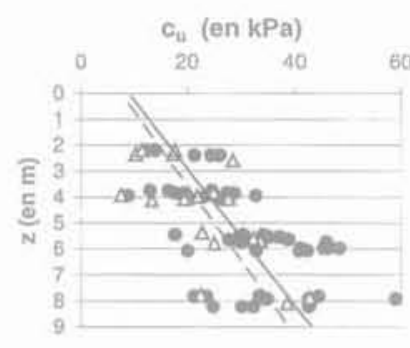

(b)

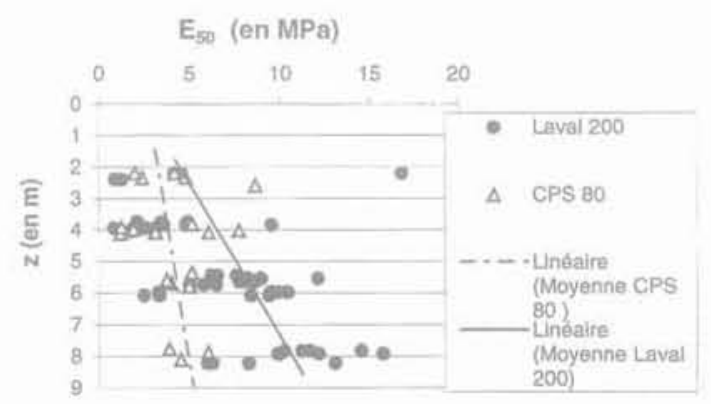

FG. 10 Variations des paramètres de déformabilité et de résistance en fonction de la profondeur pour le Laval 200 et le CPS. Strength characteristics of piston-type and Laval samplers. 


\section{Conclusion}

Ces résultats d'essais ont permis de confirmer, entre autres, quelques aspects classiques de comportement des sols compressibles déjà vérifiés sur certaines argiles molles naturelles. On observe des variations significatives de la nature des sols et de leur résistance quand on passe d'un sondage à l'autre mais aussi d'un échantillon à l'autre, ce qui n'est pas sans conséquences sur la fiabilité des conclusions que l'on peut tirer des comparaisons faites entre les différents essais. La dispersion spatiale des caractéristiques du sol sur ce site est plutôt êlevée.

Néanmoins, il apparaît que l'effet de la mise en ceuvre du carottier à piston stationnaire, notamment le fait de réaléser le forage avant un nouveau fonçage et le maintien du niveau d'eau dans le forage réduit le remaniement du sol dans les parties hautes et basses des carottes. En l'absence de réalésage, le sol subit un compactage en bas des carottes. Avant de commencer le carottage, le sol pénétrant spontanément dans le carottier, lors du déverrouillage du piston, conduit à une partie haute de l'échantillon déstructurée avec un état plutôt lâche. Si ce remplissage du carottier n'est pas pris en compte dans la longueur de fonçage, le bas de la carotte et le sol sous-jacent sont fortement comprimés et donc déstructurés. Les critères de qualité, notamment les déformations sous le poids des terres à l'œedomètre, les modules de déformation et les déformations à la rupture attestent d'une meilleure qualité des échantillons lorsque ceux-ci sont issus de sondages réalésés. Ce phénomène de remontée du sol est assez généralisé sur les chantiers ; il doit être pris en compte dans un mode opératoire du prélèvement au carottier à piston stationnaire.

Par ailleurs, on constate qu'en profondeur les prélèvements d'argile molle de Cran effectués avec le carottier Laval 200 sont moins remaniés que ceux provenant du carottier à piston stationnaire standard. Les écarts relatifs moyens entre les modules de déformation sont pratiquement de 1 à 2 . Il est primordial de choisir le matériel adéquat en fonction des objectifs de l'étude géotechnique.

\section{$\overline{\text { Bibliographie }}$}

Bat A - - Incidence des procédures de prélèvement et des procédures d'essais sur les paramètres géotechniques mesurés $x$. Thèse cie doctorat en génie civil, université de Caen, 182 p. annexes 235 p., 1999.

Hvorslev M.J. - u Subsurface exploration and sampling of soils for civil engineering purposes 10. US Army Engineers. The Waterways Experiment Station, Vicksburg. Mississippi, (available from Engineering Foundation. New York], 521 p., 1949

Idel K.H. Muhs H. Von Soos P - $\alpha$ Pror posal for quality-classes in soil sam- pling in relation to boring methods and sampling equipment 3 . Proceedings for the Speciality Session 1, "Soll Sampling $\%$. Seventh International Conference on Soil Mechanics and Foundation Engineering, Mexico City, 1969, p. 11-14.

Lacasse S., Berre T. - * Triaxial testing methods for soils „. Proc. of the Symposium on Advanced Triaxial Testing of Solls and Rocks, Louisville, Kentucky, state-of-the-art Paper, 1986, p. 264-289.

La Rochelle P. Lefebvre G. - « Sampling disturbance in Champlain clays 1. Sam- pling of Soil and Rock. American Society for Testing and Materials, STP 483, 1981, p. 143-163.

Le Masson H. Macẻ Y. - « À propos de la représentativité des échantillons préle. vés aux carottiers à piston stationnaire et par la technique du Cobra y. Bulletin de liaison des LPC. $n^{\circ} 78$, juillet-aoùt 1975, p. 83-93.

XP P 94-202 - « Sols: reconnaissance et essais. Prélèvement des sols et des roches. Méthodologie et procédures n. Norme expérimentale de l'Afnor P 94. 070, 21 p.. 1995. 